\title{
Increased expression of endothelial lipase in symptomatic and unstable carotid plaques
}

\author{
Matias Trbušić • Monika Riederer • Majda Vučić • Ivo Lovričević • \\ Božo Krušlin • Martin Gauster · Sonja Mohrenz • Andrea Berghold • \\ Beate Tiran · Vesna Degoricija $\cdot$ Saša Frank
}

Received: 15 April 2011/Revised: 24 June 2011/Accepted: 19 July 2011/Published online: 13 August 2011

(C) The Author(s) 2011. This article is published with open access at Springerlink.com

\begin{abstract}
The aim of this study was to evaluate endothelial lipase (EL) protein expression in advanced human carotid artery plaques (HCAP) with regard to plaque (in)stability and the incidence of symptoms. HCAP were collected from 66 patients undergoing carotid endarterectomy (CEA). The degree of plaque (in)stability was estimated by ultrasound and histology. In HCAP sections, EL expression was determined by immunostaining and the intensity was assessed on a semi-quantitative scale (low: $<25 \%$, high: $>25 \%$ positive cells). Monocytes and macrophages in adjacent HCAP sections were stained with a CD163 specific antibody. High EL staining was more prevalent in histologically unstable plaques (in $33.3 \%$ of fibrous plaques, $50 \%$ of ulcerated non-complicated plaques
\end{abstract}

M. Trbušić and M. Riederer are equally contributed first authors. V. Degoricija and S. Frank are equally contributed senior authors.

M. Trbušić · I. Lovričević · B. Krušlin · V. Degoricija University of Zagreb School of Medicine, Zagreb, Croatia

M. Trbušić · V. Degoricija ( ( )

Department of Medicine, Sisters of Mercy University Hospital,

Vinogradska 29, 10000 Zagreb, Croatia

e-mail: vdegoric@mef.hr

\section{Riederer $\cdot$ S. Mohrenz $\cdot$ S. Frank $(\square)$}

Institute of Molecular Biology and Biochemistry, Center of Molecular Medicine, Medical University of Graz, Harrachgasse 21/III, 8010 Graz, Austria

e-mail: sasa.frank@medunigraz.at

\section{Vučić}

University of Zagreb School of Dental Medicine,

Zagreb, Croatia

\section{Vučić · B. Krušlin}

Ljudevit Jurak University Department of Pathology,

Sisters of Mercy University Hospital, Zagreb, Croatia and $79.2 \%$ of ulcerated complicated plaques; $\chi^{2}$ test, $p=0.004)$ and in the symptomatic group (70.8 vs. $42.9 \%$ in the asymptomatic group; $\chi^{2}$ test, $\left.p=0.028\right)$. The majority of EL immunostaining was found in those HCAP regions exhibiting a strong CD163 immunostaining. EL in HCAP might be a marker and/or promoter of plaque instability and HCAP-related symptomatology.

Keywords Endothelial lipase - Carotid artery plaque . High density lipoprotein - Inflammation · Atherosclerosis . Macrophages
Abbreviations
HCAPs Human carotid artery plaques
EL Endothelial lipase
HDL High density lipoprotein
F Fibrous plaque

\section{Lovričević}

Department of Surgery, Sisters of Mercy University Hospital, Zagreb, Croatia

M. Gauster · S. Mohrenz

Institute of Cell Biology, Histology and Embryology,

Center of Molecular Medicine, Medical University of Graz,

Graz, Austria

\author{
A. Berghold \\ Institute for Medical Informatics, Statistics and Documentation, \\ Medical University of Graz, Graz, Austria \\ B. Tiran \\ Clinical Institute for Medical and Chemical Laboratory \\ Diagnostics, Medical University of Graz, Graz, Austria
}


UNC Ulcerated non-complicated

UC Ulcerated complicated

CEA Carotid endarterectomy

CDFI Color Doppler Flow Imaging

CTA Computed tomography angiography

CRP C-reactive protein

\section{Introduction}

Atherosclerosis is a chronic fibro-proliferative and immunoinflammatory disease affecting large and medium size arteries. It is characterized by endothelial dysfunction, local oxidation of circulating lipoproteins [particularly low density lipoprotein (LDL)] and their accumulation in the vascular wall [1,2], and the activation of proinflammatory cytokines [3, 4]. Inflammation plays a crucial role in plaque destabilization, as it converts stable atherosclerotic plaques, characterized by a fibrous cap covering the necrotic core, into unstable ones, characterized by a thinned fibrous cap, intra-plaque hemorrhage, surface ulceration, rupture and thrombosis [5].

Endothelial lipase (EL) is a member of the triglyceride lipase gene family, expressed by vascular endothelial cells (ECs), smooth muscle cells (SMCs) and macrophages [6, 7]. It primarily exhibits phospholipase-A1 activity, with high affinity for high density lipoprotein (HDL)-phosphatidylcholine (HDL-PC), resulting in the generation of bioactive molecules such as lysophosphatidylcholines (lyso-PC) and free fatty acids (FFA) [8]. Acting on HDL, EL not only promotes HDL remodeling but also lowering of HDL plasma levels, an independent risk factor for atherosclerosis [9]. EL plasma concentrations are strongly associated with elevated plasma interleukin-6 (IL-6), tumor necrosis factor (TNF)- $\alpha$ and C-reactive protein (CRP) levels; a profile typical of metabolic syndrome $[10,11]$. EL was also shown to promote the expression of inflammatory cytokines, exemplified by attenuation of inflammatory cytokine expression in EL-deficient macrophages [12] as well as by increased production of IL-8 in human endothelial cells over-expressing EL [13].

Taking into account the role of inflammation in plaque destabilization, together with the reported up-regulation of EL under inflammatory conditions, we assumed a positive relationship between the expression level of EL and the degree of plaque instability.

\section{Patients and methods}

Patients

Patients were recruited from the Department of Vascular Surgery, Sisters of Mercy University Hospital, Zagreb,
Croatia, between 1 November 2007 and 1 March 2009 . Written informed consent from each patient was obtained prior to the enrollment in the study, which was performed according to Good Clinical Practice and Helsinki Declaration principles. The study was approved by the local ethics committee, in accordance with institutional guidelines of the Sisters of Mercy University Hospital, Zagreb.

\section{Inclusion and exclusion criteria}

Overall, 88 patients with significant (70-99\%) carotid artery stenosis (confirmed by carotid Color Doppler Flow Imaging (CDFI) done by an experienced physician in the University Hospital) were screened for inclusion and exclusion criteria. In cases where CDFI imaging was limited by features such as calcified carotid lesions, tortuous or kinked carotid arteries or patient body constitution, contrast enhanced magnetic resonance angiography (CEMRA) or computed tomography angiography (CTA) was performed. The clinical indication for carotid endarterectomy (CEA) was met after examination by the neurologist and vascular surgeon, based on clinical presentation and CDFI (or CEMRA/CTA) results, following guidelines for treatment of extra-cranial carotid artery disease [14, 15]. Exclusion criteria were met in case of acute or chronic infection or inflammatory conditions (other than atherosclerosis), severe renal failure (serum creatinine $\geq 200 \mathrm{mmol} / \mathrm{L}$ ), severe hepatic cirrhosis (Child-Pugh Class B or C), neoplasms, recent trauma or surgery, history of ipsilateral CEA or radiotherapy and anticoagulant therapy. Patients with a possible cardiac source of embolism detected through the patients' history, electrocardiogram or echocardiography (left ventricular aneurysm, previous large anterior cardiac infarction, dilatative cardiomyopathy, aneurysm of interatrial septum with patent foramen ovale) were excluded from the study.

\section{Definition of symptomatic carotid disease}

The carotid stenosis was considered symptomatic if a patient had experienced a focal neurological symptom (ipsilateral stroke, transient ischemic attack or monocular blindness), sudden in onset and referable to the respective carotid artery within 4 months of CEA.

\section{Patient characteristics, history and medication}

Medical history was recorded from all patients, and the presence of vascular risk factors, features of metabolic syndrome, previous antihypertensive, statin, and antiplatelet treatments were noted. Clinical history was assessed for diabetes mellitus, smoking, hypertension, hyperlipidemia, prior acute myocardial infarction, symptoms of peripheral 
vascular disease and family history of CHD or IVC in firstdegree relatives under the age of 55 years. The body mass index (BMI) was calculated according to Quetelet's formula as the ratio of body weight to body height squared $\left(\mathrm{kg} / \mathrm{m}^{2}\right)$. Waist circumference was measured with a flexible tape, placed on a horizontal plane at the level of the iliac crest. Metabolic syndrome was defined according to the criteria of the International Diabetes Federation issued in 2005 (http:// www.idf.org/): increased waist circumference (for Caucasian European men $\geq 94 \mathrm{~cm}$, for women $\geq 80 \mathrm{~cm}$ ) plus any two of the following: triglycerides $>1.7 \mathrm{mmol} / \mathrm{L}$ (or treatment for elevated triglycerides), HDL cholesterol $<1.03$ $\mathrm{mmol} / \mathrm{L}$ in men or $<1.29 \mathrm{mmol} / \mathrm{L}$ in women (or treatment for low HDL), systolic blood pressure $>130 \mathrm{mmHg}$, diastolic blood pressure $>85 \mathrm{mmHg}$ (or treatment for hypertension), and fasting plasma glucose $>5.6 \mathrm{mmol} / \mathrm{L}$ (or previously diagnosed type 2 diabetes mellitus) .

Blood pressure was measured with mercurial sphygmomanometer (Riester, Jungingen, Germany) and calculated as the average of three measurements taken under standardized conditions in a supine position. Hypertension was diagnosed according to World Health Organization (WHO) criteria (blood pressure $\geq 140 / 90 \mathrm{mmHg}$ or current anti-hypertensive treatment). Diabetes was diagnosed in patients with dietary treatment, anti-diabetics or current fasting plasma glucose levels higher than $7.0 \mathrm{mmol} / \mathrm{L}$. Hyperlipoproteinaemia was defined if the low density lipoprotein (LDL) cholesterol level was $>3.5 \mathrm{mmol} / \mathrm{L}$, or if a patient was taking a lipid-lowering drug. According to cigarette smoking, patients were classified as current smokers (smoking more than five cigarettes within the past 3 months), former smokers ( $>3$ months and $<10$ years) or non-smokers.

\section{Methods}

\section{Color Doppler Flow Imaging (CDFI)}

CDFI of both carotid arteries was performed in all subjects on admission using commercially available equipment (Aloka 5,500 and Alfa 10 Premium with 10-MHz linear array transducer). The grade of plaque stenosis was assessed according to defined criteria [16]. Plaques were divided depending on the presence of the echolucency and calcification into: (1) fibrous plaques (without echolucent material and calcification), (2) soft plaques (predominantly echolucent material), (3) calcified plaques, (4) mixed plaques (both calcification and echolucent material present), and (5) non-defined plaques (fibrous plaques with small degree of echolucency and calcification). In this regard, echolucency is considered an important factor associated with symptomatic carotid disease [17] and plaque vulnerability.
Tissue sampling, preservation of carotid plaque specimens and histology

Carotid specimens were excised by the vascular surgeon (complete tubular specimen without damage to the luminal surface) and collected during surgery. They were immediately rinsed in $0.9 \%$ saline, fixed for $24 \mathrm{~h}$ in $5 \mathrm{~mL}$ buffered formalin, followed by several phosphate buffered saline (PBS) washes and storage in PBS for 2 weeks before embedding in paraffin. Plaque morphology was assessed by standard histological examination of hematoxylin and eosin-stained (HE) sections $(5 \mu \mathrm{m})$ and screened for features indicative of plaque vulnerability. Taking into account the lack of new, prospectively validated criteria for plaque vulnerability, the Stary classification [5] was applied and plaques were defined as: (1) fibrous (F) (covered by a smooth luminal surface indicative of an intact fibrous cap), (2) ulcerated non-complicated (UNC) (thin fissured or ruptured cap), and (3) ulcerated complicated (UC) (thin fissured or ruptured cap with intra-plaque hemorrhage and/or thrombus).

\section{Immunohistochemistry}

Formalin fixed and paraffin embedded human carotid sections $(5 \mu \mathrm{m})$ were mounted on Superfrost Plus slides (Menzel, Braunschweig, Germany) and dried at $40^{\circ} \mathrm{C}$ overnight. Slides were dewaxed twice in xylene for $10 \mathrm{~min}$ and rehydrated through a graded series of alcohol. Antigen retrieval was performed by sequentially incubating the slides in antigen retrieval buffer $\mathrm{pH} 9$ (Eubio, Vienna Austria) for $2 \mathrm{~min}$ at $720 \mathrm{~W}$ and $3 \times 5 \mathrm{~min}$ at $160 \mathrm{~W}$ in the microwave. Immunohistochemistry was performed using the Ultravision LP detection system (Thermo Scientific, Fremont, USA) according to the manufacturer's instructions. In brief, endogenous peroxidase was blocked by incubation with Hydrogen Peroxide Block (Thermo Scientific) for $10 \mathrm{~min}$. Thereafter, slides were washed with PBS and unspecific background was blocked with Ultra V Block (Thermo scientific) for 8 min. Rabbit clonal antihuman CD163 (DB Biotech, clone K20-T, diluted 1:100) and rabbit anti-EL serum $(1: 1,500)$ [18] were diluted in antibody diluent (DAKO) and incubated on slides for $1 \mathrm{~h}$ at RT. After PBS washing steps, detection was achieved by incubation with the UltraVision HRP-labelled polymer system for $15 \mathrm{~min}$ and 3-amino-9-ethylcarbacole (AEC, Dako, Denmark), according to the manufacturer's instructions. For negative controls, slides were incubated with rabbit IgG Ab-1 $(2 \mu \mathrm{g} / \mathrm{ml}$, Neomarkers $)$ instead of the primary antibodies. Nuclei were stained with hemalaun and slides were mounted with Kaiser's glycerol gelatine. Images of sections were taken with an Axiophot microscope equipped with an AxioCam HRc digital camera (Zeiss, 
Oberkochen, Germany). Importantly, all samples were processed simultaneously using the same batch of reagents. The expression of EL was evaluated on a semi-quantitative scale by three independent, experienced investigators blinded to the clinical and histology data. The scoring was arbitrary, as follows: (1) low staining (A) $(<25 \%$ positive cells), and (2) high staining (B) ( $>25 \%$ positive cells).

Assays

Blood samples for laboratory assays were obtained before surgery at approximately 8 am, following overnight fasting. Blood sampled in ethylenediaminetetraacetic acid (EDTA)-K3 containing tubes was used for hematological assays (Coulter-Counter S plus junior, Coulter Electornics Limited, Luton, UK). Coagulation parameters were assessed from blood samples in $3.8 \%$ sodium citrate. After separation of plasma by centrifugation ( $3000 \mathrm{~g}$ for $10 \mathrm{~min}$ ), the Quicktest was performed on a Behring coagulation timer (Dade Behring, Marburg, Germany). Serum creatinine, bilirubin, aspartate transaminase, alanine transaminase, blood glucose level, total plasma cholesterol, triglyceride, LDL and HDL were measured on automated, multi-channel selective analyzers Olympus AU2700 and Olympus Fractoscan junior (Olympus Diagnostica GmbH, Hamburg, Germany). High sensitivity (hs) CRP concentrations were measured on automated, multichannel selective analyzer Modular (Roche Diagnostics, Mannheim, Germany). IL-6 concentrations were measured using a specific chemiluminescent ELISA (QuantiGlo; R\&D Systems, Wiesbaden-Nordenstadt, Germany) according to the manufacturers' instructions.

\section{Statistical analysis}

Data were summarized using mean and standard deviation or median, minimum, maximum as appropriate for continuous variables and counts as well as percentages for categorical variables. Data distribution was analyzed by Kolmogorov-Smirnov test. According to these results, appropriate parametric or non-parametric tests were used in further analyses. Unpaired Student's $t$-test or MannWhitney $U$ test was performed to assess differences among quantitative variables between symptomatology and immunohistochemistry groups. Differences between ordinally scaled histological groups were analyzed with Kruskal-Wallis test, whereas Chi square test was applied to analyze differences in qualitative and categorical variables. The association between quantitative data was evaluated using Spearman-rank correlation analysis. Data were analyzed using PASW 17.02 (IBM, Chicago, IL, USA) software and all $p$ values below 0.05 were considered significant.

\section{Results}

Sample size and clinical characteristics

Out of 88 patients assessed for eligibility, 22 patients were excluded (see Fig. 1). The final study population included 66 patients (43 males and 23 females, mean age 67.5 years) stratified into two groups: the asymptomatic group (42 pts) and the symptomatic group ( $24 \mathrm{pts})$. The patients' clinical characteristics and medications are shown in Table 1. The two groups did not differ significantly according to age, gender, individual risk factors, medication, or coexistence of other vascular diseases (peripheral vascular disease, past myocardial infarction, angina pectoris). In the symptomatic group, however, a higher incidence of patients with increased waist circumference was found ( 62.5 vs. $38 \% ; \chi^{2}$ test, $p=0.049$ ). The BMI was also higher in the symptomatic group $(28.2 \pm 3.0$ vs. $26.8 \pm 3.0)$ but the difference did not reach statistical significance $(p=0.085)$. Overall, there was no significant difference in the number of metabolic risk factors. Basic biochemical and hematological parameters as well as inflammatory parameters (WBC, hs-CRP, erythrocyte sedimentation rate, fibrinogen and IL-6) did not differ between the groups (Table 2).

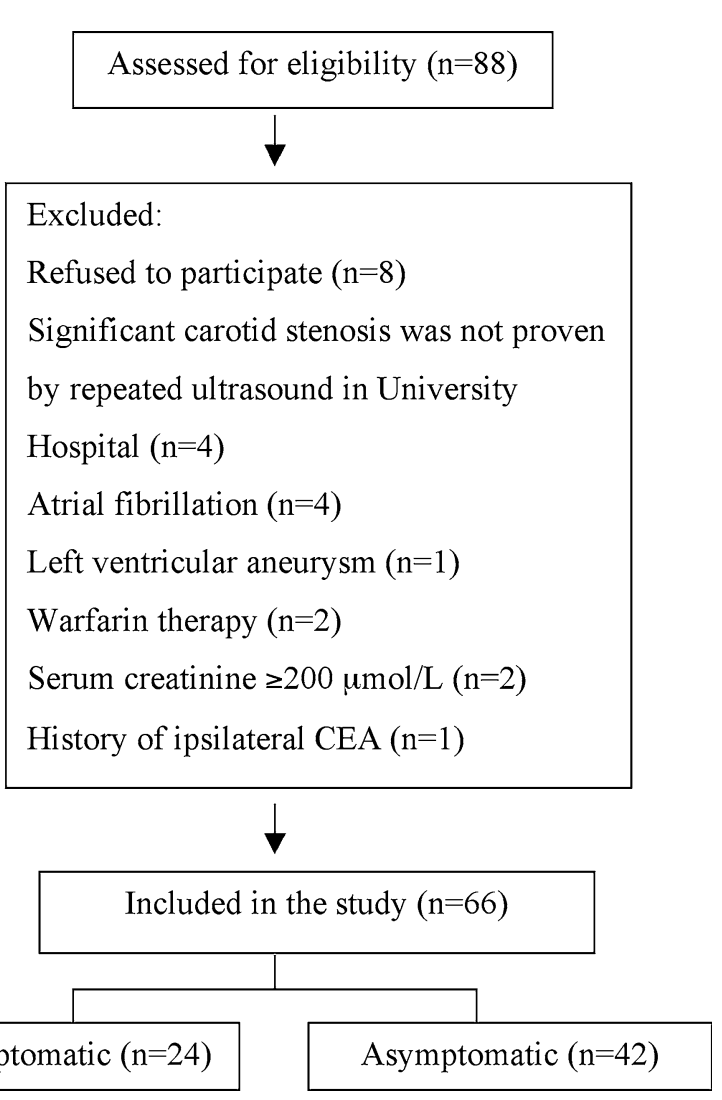

Fig. 1 Flow chart of the study 
Table 1 Clinical characteristics and patient medications at the enrollment into the study
$B M I$ body mass index, $A C E$ angiotensin-converting enzyme

${ }^{a}$ Familiar history of cardiovascular disease or stoke in first relatives younger than 55 years

${ }^{\dagger}$ Fisher exact test, $p=0.049$
Clinical characteristic and medications

Asymptomatic $(n=42)$

$($ mean $\pm \mathrm{SD})$

Symptomatic $(n=24)$

$($ mean $\pm \mathrm{SD})$

\begin{tabular}{|c|c|c|}
\hline Age (years; median, range) & $69(43-83)$ & $65.5(50-84)$ \\
\hline $\mathrm{BMI}\left(\mathrm{kg} / \mathrm{m}^{2} ;\right.$ mean $\left.\pm \mathrm{SD}\right)$ & $26.8 \pm 3.0$ & $28.2 \pm 3.0$ \\
\hline \multirow[t]{2}{*}{ Waist circumference $(\mathrm{cm}$; mean $\pm \mathrm{SD})$} & $91.6 \pm 7.51$ & $94.1 \pm 8.5$ \\
\hline & \multicolumn{2}{|c|}{ No. of patients $(\%)$} \\
\hline \multicolumn{3}{|l|}{ Gender } \\
\hline Men & $28(66.6)$ & $15(62.5)$ \\
\hline Women & $14(33.3)$ & $9(37.5)$ \\
\hline Increased waist circumference $^{\dagger}$ & $16(38.1)$ & $15(62.5)$ \\
\hline \multicolumn{3}{|l|}{ Cardiovascular risk factors } \\
\hline Arterial hypertension & $36(85.7)$ & $22(91.6)$ \\
\hline Hyperlipoproteinaemia & $31(73.8)$ & $21(87.5)$ \\
\hline Diabetes mellitus or glucose intolerance & $20(47.6)$ & $15(62.5)$ \\
\hline Tobacco (current or recent) & $16(38.0)$ & $14(58.3)$ \\
\hline Family history $^{\mathrm{a}}$ & $9(21.4)$ & $4(16.7)$ \\
\hline Peripheral vascular disease & $21(50.0)$ & $9(37.5)$ \\
\hline Coronary artery disease & $12(28.5)$ & $6(25.0)$ \\
\hline \multicolumn{3}{|l|}{ Medications } \\
\hline Statins & $29(69.0)$ & $16(66.6)$ \\
\hline ACE inhibitors & $21(50.0)$ & $12(50.0)$ \\
\hline Angiotensin receptor blockers & 7 (16.6) & $3(12.5)$ \\
\hline Calcium channel blockers & $19(45.2)$ & $8(33.3)$ \\
\hline Acetyl salicylic acid & $25(59.2)$ & $19(79.1)$ \\
\hline \multicolumn{3}{|l|}{ No. of metabolic elements present } \\
\hline 0 & $3(7.1)$ & $1(4.2)$ \\
\hline 1 & $4(9.5)$ & $2(8.3)$ \\
\hline 2 & $15(35.7)$ & $5(20.8)$ \\
\hline 3 & $8(19.0)$ & $4(16.7)$ \\
\hline 4 & $9(21.4)$ & $11(45.8)$ \\
\hline 5 & $3(7.1)$ & $1(4.2)$ \\
\hline
\end{tabular}

Ultrasound and histological characteristics of plaques

The grade of carotid artery stenosis detected by CDFI (or CEMRA/CTA) was considerably higher in the symptomatic group ( $\chi^{2}$ test, $\left.p=0.027\right)$ (Table 3 ). Additionally, plaques with morphologic characteristics suggestive of plaque vulnerability (echolucent zones with or without ulcerated morphology) seen on CDFI were more frequent in the symptomatic group ( $\chi^{2}$ test, $p=0.039$ ). Finally, the majority of asymptomatic plaques $(50 \%)$ were $\mathrm{F}$ plaques, whereas the majority of symptomatic plaques $(50 \%)$ were UC plaques (not statistically significant) (Table 3 ).

\section{EL immunostaining in plaques}

In $\mathrm{F}$ plaques (Fig 2a-c), the necrotic core was covered with a fibrotic cap with preserved endothelium and only few inflammatory cells (Fig. 2a). In UC plaques (Fig. 2d-e), in contrast, the necrotic core was covered with inflammatory infiltrates, composed of lymphocytes, macrophages, siderophages and importantly of erythrocytes, indicating intraplaque hemorrhage (Fig 2d). In F plaques, a weak EL immunostaining (type A) was detected in the endothelial layer and in the fibrous cap (Fig. 2b). The fibrous cap also exhibited moderate CD163 immunostaining, indicative of the presence of monocytes and macrophages (Fig. 2c). Compared with $\mathrm{F}$ plaques, EL staining was more pronounced (type B) in UC plaques (Fig. 2e). Here, EL staining localized primarily to cells of the inflammatory infiltrate (Fig. 2e), which showed a strong staining for CD163 as well (Fig. 2f).

Importantly, high intensity EL immunostaining (type B) was most frequently observed in UC plaques $(79 \% ; 19$ of 24 pts), followed by UNC and F with 50\% (6 of 12 pts) and $33 \%$ (10 of 30 pts), respectively (Fig. 3) ( $\chi^{2}$ test, $p=0.004)$. Furthermore, EL immunostaining type B was 
Table 2 Patient blood parameters at the enrollment in the study

\begin{tabular}{lll}
\hline Parameter & $\begin{array}{l}\text { Asymptomatic } \\
(n=42) \\
(\text { mean } \pm \mathrm{SD})\end{array}$ & $\begin{array}{l}\text { Symptomatic } \\
(n=24) \\
(\text { mean } \pm \mathrm{SD})\end{array}$ \\
\hline Platelets $\left(\times 10^{9} / \mathrm{L}\right)$ & $237 \pm 67$ & $219 \pm 52$ \\
Hemoglobin $(\mathrm{g} / \mathrm{L})$ & $137.8 \pm 14.1$ & $140.5 \pm 9.1$ \\
WBC $\left(\times 10^{9} / \mathrm{L}\right)$ & $8.21 \pm 2.4$ & $7.33 \pm 1.65$ \\
Fibrinogen $(\mathrm{g} / \mathrm{L})$ & $4.28 \pm 0.86$ & $4.62 \pm 1.02$ \\
APTT $(\mathrm{s})$ & $25.2 \pm 4.6$ & $26.4 \pm 3.4$ \\
PT $(\%)$ & $104.3 \pm 11.8$ & $103.6 \pm 8.9$ \\
ESR $(\mathrm{mm} / \mathrm{h})$ & $18.5 \pm 8.7$ & $19.2 \pm 11.2$ \\
hs-CRP $(\mu \mathrm{g} / \mathrm{mL})$ & $1.4(0.1-23.0)^{\mathrm{a}}$ & $3.6(0.4-31.5)^{\mathrm{a}}$ \\
Serum creatinine $(\mu \mathrm{mol} / \mathrm{L})$ & $95.1 \pm 21.7$ & $96.7 \pm 20.4$ \\
Total cholesterol $(\mathrm{mmol} / \mathrm{L})$ & $5.2 \pm 1.5$ & $5.5 \pm 1.4$ \\
LDL $(\mathrm{mmol} / \mathrm{L})$ & $3.1 \pm 1.3$ & $3.5 \pm 1.3$ \\
HDL $(\mathrm{mmol} / \mathrm{L})$ & $1.3 \pm 0.4$ & $1.3 \pm 0.3$ \\
Triglycerides $(\mathrm{mmol} / \mathrm{L})$ & $1.8 \pm 0.7$ & $1.8 \pm 0.9$ \\
Lp(a) $(\mathrm{mg} / \mathrm{dl})$ & $23.4 \pm 21.2$ & $19.9 \pm 18.7$ \\
IL-6 $(\mathrm{pg} / \mathrm{ml})$ & $1.4(0.0-15.4)^{\mathrm{a}}$ & $1.8(0.0-43.3)^{\mathrm{a}}$
\end{tabular}

WBC White blood cells, APTT activated partial thromboplastin time, $P T$ prothrombin time, ESR erythrocyte sedimentation rate at first hour, $h s-C R P$ high sensitivity C-reactive protein, $L D L$ low density lipoprotein, $H D L$ high density lipoprotein, $I L-6$ interleukin-6, $L p(a)$ Lipoprotein (a)

${ }^{\mathrm{a}}$ Results are median (range)

significantly more prevalent in plaques of symptomatic patients (Table 3$)\left(\chi^{2}\right.$ test, $\left.p=0.028\right)$. Inflammatory markers (IL-6 and hsCRP) as well as HDL plasma levels were comparable between patients with EL immunostaining type A and B (Mann-Whitney $U$ test, $p=0.17$ for IL$6 ; p=0.44$ for hsCRP; $p=0.76$ for HDL).

\section{Discussion}

The major findings of the present study are: (1) higher EL immunostaining in unstable HCAP and (2) higher EL immunostaining in plaques of patients exhibiting HCAPrelated symptomatology. Probably the main connector between plaque stability, inflammation and EL are macrophages, which play an important role in plaque destabilization [19]. Using immunohistochemistry, Azumi et al. [20] identified macrophages and smooth muscle cells as main source of EL in HCAP. This was confirmed by a Danish study [21], which detected EL mRNA and protein in areas between the fibrotic cap and the necrotic core, colocalizing primarily with macrophage specific CD68; however, that study exclusively included symptomatic carotid patients ( $26 \mathrm{pts}$ ). In the present study, the intensity of both EL and CD163 immunostaining increased with plaque instability, however not all CD163 positive regions/
Table 3 Ultrasound and histological characteristics of carotid stenosis

\begin{tabular}{|c|c|c|}
\hline \multirow[t]{2}{*}{ Parameter } & \multicolumn{2}{|c|}{ No. of patients (\%) (total $n=66$ ) } \\
\hline & $\begin{array}{l}\text { Asymptomatic } \\
(n=42)\end{array}$ & $\begin{array}{l}\text { Symptomatic } \\
(n=24)\end{array}$ \\
\hline \multicolumn{3}{|c|}{ Grade of stenosis $(\%)^{\dagger}$} \\
\hline $70-85$ & $18(42.9)$ & $4(16.7)$ \\
\hline $85-95$ & $5(11.9)$ & $1(4.2)$ \\
\hline $95-99$ & $19(45.2)$ & $19(79.2)$ \\
\hline \multicolumn{3}{|c|}{ Morphology of plaques by ultrasound } \\
\hline Fibrous & $5(11.9)$ & $0(0.0)$ \\
\hline Soft & $11(26.2)$ & $12(50.0)$ \\
\hline Calcified & $4(9.5)$ & $3(12.5)$ \\
\hline Mixed & $14(33.3)$ & $9(37.5)$ \\
\hline Non-defined & $8(19.0)$ & $0(0.0)$ \\
\hline \multicolumn{3}{|c|}{ Histology classification } \\
\hline Fibrous & $21(50.0)$ & $9(37.5)$ \\
\hline UNC & $9(21.4)$ & $3(12.5)$ \\
\hline UC & $12(28.6)$ & $12(50.0)$ \\
\hline \multicolumn{3}{|c|}{ Immunohistochemistry for $\mathrm{EL}^{\S}$} \\
\hline A $(0-25 \%$ cells $)$ & $24(57.1)$ & $7(29.2)$ \\
\hline $\mathrm{B}(>25 \%$ cells $)$ & $18(42.9)$ & $17(70.8)$ \\
\hline
\end{tabular}

$U N C$ ulcerated non-complicated plaques (without intra-plaque hemorrhage and/or thrombus), $U C$ ulcerated complicated (with intraplaque hemorrhage and/or thrombus), EL-endothelial lipase

${ }^{\dagger} \chi^{2}, p=0.027$

* $\chi^{2}, p=0.039$

$\S \chi^{2}, p=0.028$

cells were positive for EL staining. This might be explained by the fact that CD163 (a scavenger receptor for haptoglobin/hemoglobin complexes) is expressed by both monocytes and macrophages [22], whereas EL protein expression is negligible in monocytes but prominent in macrophages and foam cells [21]. Accordingly, the upregulation of EL (in plaque macrophages) might aggravate inflammation by promoting cytokine production $[12,13]$ or LDL uptake [23, 24].

Most studies, addressing EL in the context of inflammation and atherosclerosis, were based on the plasma levels of EL. In healthy subjects with a family history of premature coronary heart disease (CHD), increased plasma EL concentrations were associated with subclinical CHD [10]. Badelino et al. [25] reported increased plasma EL levels under experimental endotoxemia and in patients with metabolic syndrome, a known proinflammatory condition.

In the present study, where all patients exhibited advanced atherosclerotic lesions in at least one (target) artery, inflammatory serum markers, such as IL-6 and hsCRP, were not different between patients with low and high EL expression in HCAP (Mann-Whitney $U$ test, 

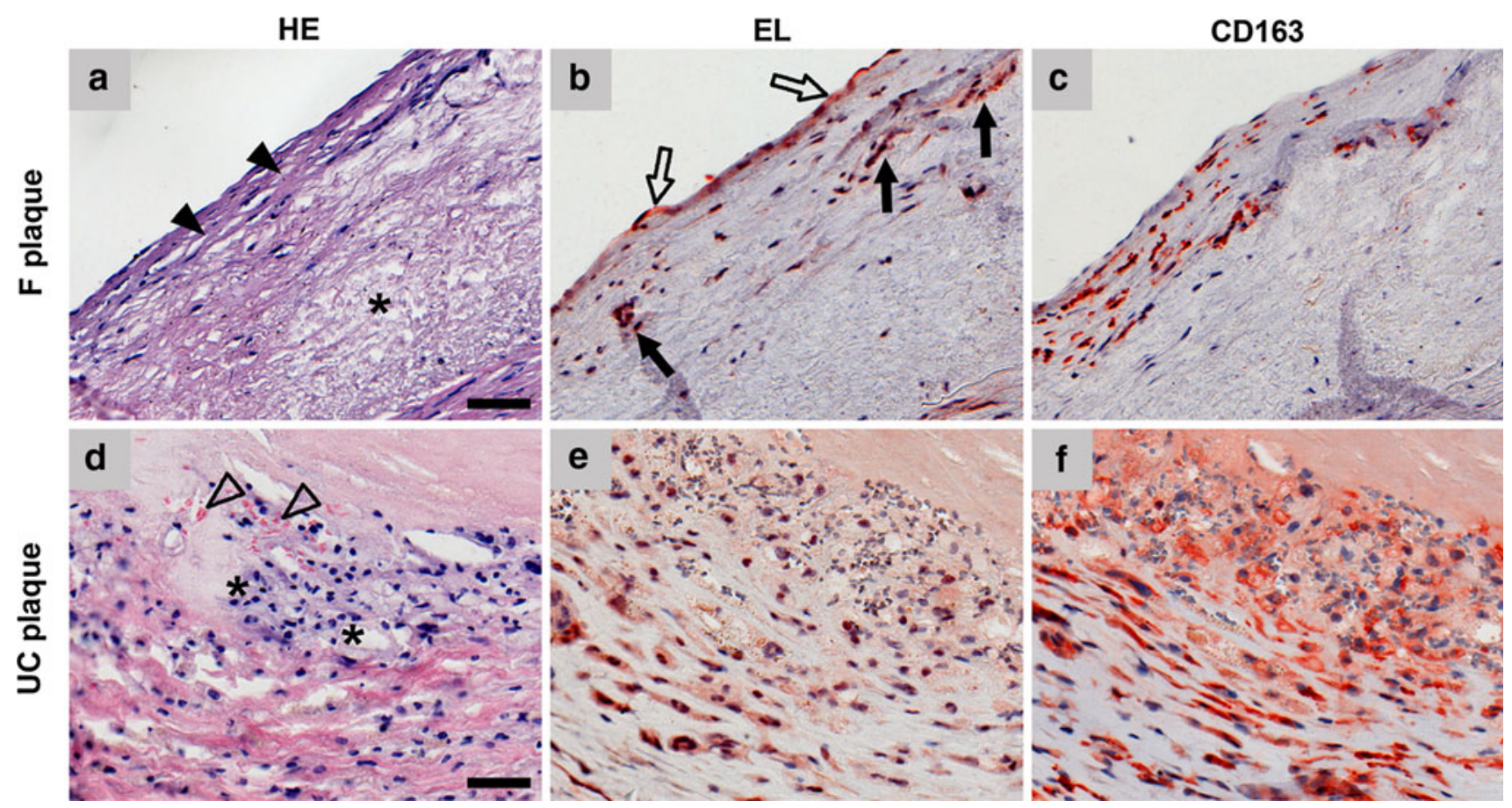

Fig. 2 HCAP histology and immunostaining of EL and CD163. For histology, serial sections of HCAP were stained with HE and for immunohistochemical detection of EL and CD163, with antibodies against EL and the mononocyte/macrophage marker CD163. A fibrous plaque $(\mathrm{F})$ is shown in panels a-c: a HE-stained $\mathrm{F}$ plaque with fibrous cap (filled arrow heads) and necrotic core (asterisk). b EL

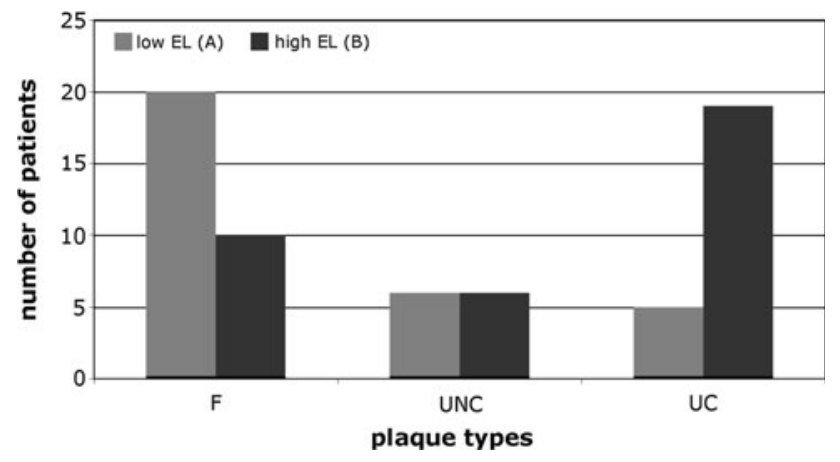

Fig. 3 The intensity of EL immunostaining increases with plaque instability. The intensity of EL immunostaining was determined in different types of plaques, defined by histology as fibrous plaques $(\mathrm{F})$, ulcerated non-complicated plaques (UNC), and ulcerated complicated plaques (UC). Grey columns represent the number of patients exhibiting low intensity of EL immunostaining $(A)$, whereas black columns denote those with high intensity of EL immunostaining $(B)$ in plaques. $79.2 \%$ of UC plaques were type B, $50 \%$ of UNC plaques were type $\mathrm{B}$, whereas $66.7 \%$ of $\mathrm{F}$ plaques exhibited type $\mathrm{A}$ immunostaining. $\chi^{2}$ test, $p=0.004$

$p=0.17$ for IL-6; $p=0.44$ for hsCRP). This might probably reflect the diffuse nature of atherosclerosis that masks the effect of increased local inflammatory molecule production in unstable HCAP, highlighting the importance and relevance of localized EL expression at the site of staining (red) in the endothelium (empty arrows) and in cells within the fibrous cap (filled arrows). c CD163 immunostaining (red). An ulcerated plaque (UC) is shown in panels d-f: d HE-stained UC plaque with inflammatory infiltrate (asterisks) and intra-plaque erythrocytes (empty arrow heads). e EL immunostaining. f CD163 staining. Scale bar represents $50 \mu \mathrm{m}$

pathology. Furthermore, this lack of association between local EL expression in HCAP and IL-6 and hsCRP might be due to the analysis of the total cohort irrespective of gender [26], age [27], or routine exercise [28]—factors with established impact on the plasma levels of inflammatory molecules. Additionally, the expression of inflammatory molecules might have been differently affected by medication-considering pharmacological properties, dosage and interactions of the therapeutics, and the duration of the treatment.

In the present study, there was no significant difference in plasma HDL levels between patients with high (B) and low (A) EL expression in plaques (mean value in both groups $1.3 \mathrm{mmol} / \mathrm{L}$, Mann-Whitney $U$ test, $p=0.76$ ). Importantly, in the study group, $69 \%$ of patients were treated with statins (atorvastatin 19 pts, simvastatin 23 pts, fluvastatin 3 pts) known to increase HDL plasma levels by regulating various molecules that participate in reverse cholesterol transport, such as apo AI [29], scavenger receptor B type I (SR-BI) [30], or ATP binding cassette A1 (ABCA1) [31]. Furthermore, statins were shown to decrease EL expression [32, 33]. Considering this and the fact that patients were treated with various doses of different statins, known to vary in their potency to modulate HDL plasma levels, it is tempting to assume that the lack of 
association between EL in HCAP and HDL plasma levels in the present study is partially due to medication. In addition to statins, angiotensinogen II (AT II) receptor antagonists (10 pts), as well as angiotensin converting enzyme inhibitors (33 pts) might, considering the capacity of AT II to modulate EL expression [34], affect the relationship between EL in HCAP and HDL plasma levels as well as between EL in HCAP and inflammatory markers. Interestingly, neither statins nor ACE inhibitors impacted significantly the intensity of EL immunostaining in HCAP (not shown). Furthermore, increased EL mass in HCAP does not necessarily have to be accompanied by increased local EL phospholipase activity, considering reduced phospholipase activity of various genetic variants [35]. Finally, the relative contribution of EL in HCAP to the total systemic EL phospholipase activity might be too low to significantly affect HDL plasma levels. Besides, even serum EL levels were found not to correlate significantly with HDL levels [11, 36].

The results of our study are in line with previous reports showing no obligatory association between symptomatology of carotid disease and histological features of plaque vulnerability. This might be due to: (1) the lack of strong uniform criteria for plaque instability, (2) artificial (laboratory) disruption of the plaque surface, or iii) some unstable plaques which resolve spontaneously without causing symptoms [37-39]. In addition, some of the ischemic events are not caused by the carotid lesion itself, but rather by embolic material derived from heart and aorta. Using echocardiography, we could exclude only some of the patients with suspected cardiac source of embolism. Moreover, it is known that not only the vulnerable plaque makes the patient vulnerable, but also that the vulnerable environment (e.g., blood prone to thrombosis) plays an important role in the overall outcome.

To our knowledge this is the first study addressing the relationship between the intensity of EL immunostaining in HCAP and the degree of HCAP vulnerability and HCAPrelated symptomatology. The higher EL immunostaining in vulnerable and symptomatic HCAP suggests a strong involvement of EL in the local inflammatory process, a key element of plaque destabilization. Whether EL in HCAP is only a marker or an active causative factor for cerebrovascular events and whether EL in HCAP has a prognostic value for the incidence and velocity of carotid artery restenosis or other cerebro- and/or cardio-vascular events remains to be examined.

Acknowledgments This work was supported by the Austrian Science Foundation (FWF; grant P19473-B05 to S.F.), the Jubilee Foundation of the Austrian National Bank (grant 12778 to S.F.), the Lanyar Foundation (grant 328 to S.F.) and Land Steiermark (grant to M.R.). We thank Margarete Lechleitner and Jasna Matic for expert technical assistance.
Conflict of interest Authors declare that they have no conflict of interest.

Open Access This article is distributed under the terms of the Creative Commons Attribution Noncommercial License which permits any noncommercial use, distribution, and reproduction in any medium, provided the original author(s) and source are credited.

\section{References}

1. Libby P, Ridker PM, Maseri A (2002) Inflammation and atherosclerosis. Circulation 105(9):1135-1143

2. Hansson GK (2005) Inflammation, atherosclerosis, and coronary artery disease. N Engl J Med 352(16):1685-1695. doi:10.1056/ NEJMra043430

3. Fuster V, Moreno PR, Fayad ZA, Corti R, Badimon JJ (2005) Atherothrombosis and high-risk plaque: part I: evolving concepts. J Am Coll Cardiol 46(6):937-954. doi:10.1016/j.jacc.2005.03.074

4. Schieffer B, Selle T, Hilfiker A, Hilfiker-Kleiner D, Grote K, Tietge UJ, Trautwein C, Luchtefeld M, Schmittkamp C, Heeneman S, Daemen MJ, Drexler H (2004) Impact of interleukin-6 on plaque development and morphology in experimental atherosclerosis. Circulation 110(22):3493-3500

5. Stary HC, Chandler AB, Dinsmore RE, Fuster V, Glagov S, Insull W Jr, Rosenfeld ME, Schwartz CJ, Wagner WD, Wissler RW (1995) A definition of advanced types of atherosclerotic lesions and a histological classification of atherosclerosis. A report from the committee on vascular lesions of the council on arteriosclerosis, American Heart Association. Arterioscler Thromb Vasc Biol 15(9):1512-1531

6. Hirata K, Dichek HL, Cioffi JA, Choi SY, Leeper NJ, Quintana L, Kronmal GS, Cooper AD, Quertermous T (1999) Cloning of a unique lipase from endothelial cells extends the lipase gene family. J Biol Chem 274(20):14170-14175

7. Jaye M, Lynch KJ, Krawiec J, Marchadier D, Maugeais C, Doan K, South V, Amin D, Perrone M, Rader DJ (1999) A novel endothelial-derived lipase that modulates HDL metabolism. Nat Genet 21(4):424-428

8. Gauster M, Rechberger G, Sovic A, Horl G, Steyrer E, Sattler W, Frank S (2005) Endothelial lipase releases saturated and unsaturated fatty acids of high density lipoprotein phosphatidylcholine. J Lipid Res 46(7):1517-1525. doi:10.1194/j1r.M500054-JLR200

9. Yasuda T, Ishida T, Rader DJ (2010) Update on the role of endothelial lipase in high-density lipoprotein metabolism, reverse cholesterol transport, and atherosclerosis. Circ J 74(11):22632270 (JST.JSTAGE/circj/CJ-10-0934)

10. Badellino KO, Wolfe ML, Reilly MP, Rader DJ (2006) Endothelial lipase concentrations are increased in metabolic syndrome and associated with coronary atherosclerosis. PLoS Med/Public Library of Science 3(2):e22

11. Paradis ME, Badellino KO, Rader DJ, Deshaies Y, Couture P, Archer WR, Bergeron N, Lamarche B (2006) Endothelial lipase is associated with inflammation in humans. J Lipid Res 47(12): 2808-2813

12. Qiu G, Ho AC, Yu W, Hill JS (2007) Suppression of endothelial or lipoprotein lipase in THP-1 macrophages attenuates proinflammatory cytokine secretion. J Lipid Res 48(2):385-394. doi: 10.1194/jlr.M600304-JLR200

13. Riederer M, Lechleitner M, Hrzenjak A, Koefeler H, Desoye G, Heinemann A, Frank S (2011) Endothelial lipase (EL) and EL-generated lysophosphatidylcholines promote IL-8 expression in endothelial cells. Atherosclerosis 214(2):338-344. doi: 10.1016/j.atherosclerosis.2010.11.007 
14. Anonymous (1995) Endarterectomy for asymptomatic carotid artery stenosis. Executive committee for the asymptomatic carotid atherosclerosis study. JAMA 273(18):1421-1428

15. Barnett HJ, Taylor DW, Eliasziw M, Fox AJ, Ferguson GG, Haynes RB, Rankin RN, Clagett GP, Hachinski VC, Sackett DL, Thorpe KE, Meldrum HE, Spence JD (1998) Benefit of carotid endarterectomy in patients with symptomatic moderate or severe stenosis. North american symptomatic carotid endarterectomy trial collaborators. N Engl J Med 339(20):1415-1425

16. Lovrencic-Huzjan A, Strineka M, Aiman D, Strbe S, SodecSimicevic D, Demarin V (2009) The contralateral carotid disease in patients with internal carotid artery occlusion. Acta Clin Croat 48(3):241-246

17. Giannoukas AD, Sfyroeras GS, Griffin M, Saleptsis V, Antoniou GA, Nicolaides AN (2009) Association of plaque echostructure and cardiovascular risk factors with symptomatic carotid artery disease. Vasa 38(4):357-364. doi:10.1024/0301-1526.38.4.357

18. Gauster M, Hiden U, Blaschitz A, Frank S, Lang U, Alvino G, Cetin I, Desoye G, Wadsack C (2007) Dysregulation of placental endothelial lipase and lipoprotein lipase in intrauterine growthrestricted pregnancies. J Clin Endocrinol Metab 92(6):2256-2263

19. Alsheikh-Ali AA, Kitsios GD, Balk EM, Lau J, Ip S (2010) The vulnerable atherosclerotic plaque: scope of the literature. Ann Intern Med 153(6):387-395. doi:10.1059/0003-4819-153-6201009210-00272

20. Azumi H, Hirata K, Ishida T, Kojima Y, Rikitake Y, Takeuchi S, Inoue N, Kawashima S, Hayashi Y, Itoh H, Quertermous T, Yokoyama M (2003) Immunohistochemical localization of endothelial cell-derived lipase in atherosclerotic human coronary arteries. Cardiovasc Res 58(3):647-654 (S0008636303002876)

21. Bartels ED, Nielsen JE, Lindegaard ML, Hulten LM, Schroeder TV, Nielsen LB (2007) Endothelial lipase is highly expressed in macrophages in advanced human atherosclerotic lesions. Atherosclerosis 195(2):e42-e49. doi:10.1016/j.atherosclerosis.2007. 05.002

22. Schaer DJ, Alayash AI, Buehler PW (2007) Gating the radical hemoglobin to macrophages: the anti-inflammatory role of CD163, a scavenger receptor. Antioxid Redox Signal 9(7):991999. doi:10.1089/ars.2007.1576

23. Qiu G, Hill JS (2007) Endothelial lipase enhances low density lipoprotein binding and cell association in THP-1 macrophages. Cardiovasc Res 76(3):528-538

24. Yasuda T, Hirata K, Ishida T, Kojima Y, Tanaka H, Okada T, Quertermous T, Yokoyama M (2007) Endothelial lipase is increased by inflammation and promotes LDL uptake in macrophages. J Atheroscler Thromb 14(4):192-201 (JST.JSTAGE/jat/ E502)

25. Badellino KO, Wolfe ML, Reilly MP, Rader DJ (2008) Endothelial lipase is increased in vivo by inflammation in humans. Circulation 117(5):678-685. doi:10.1161/CIRCULATIONAHA. 107.707349

26. van Eijk LT, Dorresteijn MJ, Smits P, van der Hoeven JG, Netea MG, Pickkers P (2007) Gender differences in the innate immune response and vascular reactivity following the administration of endotoxin to human volunteers. Crit Care Med 35(6):1464-1469. doi:10.1097/01.CCM.0000266534.14262.E8

27. Maggio M, Basaria S, Ceda GP, Ble A, Ling SM, Bandinelli S, Valenti G, Ferrucci L (2005) The relationship between testosterone and molecular markers of inflammation in older men. J Endocrinol Invest 28(11 Suppl Proceedings):116-119

28. Roberts CK, Won D, Pruthi S, Lin SS, Barnard RJ (2006) Effect of a diet and exercise intervention on oxidative stress, inflammation and monocyte adhesion in diabetic men. Diabetes Res Clin Pract 73(3):249-259. doi:10.1016/j.diabres.2006.02.013

29. Noji Y, Higashikata T, Inazu A, Nohara A, Ueda K, Miyamoto S, Kajinami K, Takegoshi T, Koizumi J, Mabuchi H (2002) Longterm treatment with pitavastatin (NK-104), a new HMG-CoA reductase inhibitor, of patients with heterozygous familial hypercholesterolemia. Atherosclerosis 163(1):157-164 (S002191 5001007651)

30. Han J, Parsons M, Zhou X, Nicholson AC, Gotto AM Jr, Hajjar DP (2004) Functional interplay between the macrophage scavenger receptor class B type I and pitavastatin (NK-104). Circulation 110(22):3472-3479. doi:10.1161/01.CIR.0000148368.79202.F1

31. Zanotti I, Favari E, Sposito AC, Rothblat GH, Bernini F (2004) Pitavastatin increases ABCA1-mediated lipid efflux from Fu5AH rat hepatoma cells. Biochem Biophys Res Commun 321(3):670 674. doi:10.1016/j.bbrc.2004.07.020

32. Kojima Y, Ishida T, Sun L, Yasuda T, Toh R, Rikitake Y, Fukuda A, Kume N, Koshiyama H, Taniguchi A, Hirata K (2010) Pitavastatin decreases the expression of endothelial lipase both in vitro and in vivo. Cardiovasc Res 87(2):385-393. doi:10.1093/ cvr/cvp419

33. Qiu G, Hill JS (2007) Atorvastatin decreases lipoprotein lipase and endothelial lipase expression in human THP-1 macrophages. J Lipid Res 48(10):2112-2122

34. Shimokawa Y, Hirata K, Ishida T, Kojima Y, Inoue N, Quertermous T, Yokoyama M (2005) Increased expression of endothelial lipase in rat models of hypertension. Cardiovasc Res 66(3):594-600. doi:10.1016/j.cardiores.2005.01.013

35. Edmondson AC, Brown RJ, Kathiresan S, Cupples LA, Demissie S, Manning AK, Jensen MK, Rimm EB, Wang J, Rodrigues A, Bamba V, Khetarpal SA, Wolfe ML, Derohannessian S, Li M, Reilly MP, Aberle J, Evans D, Hegele RA, Rader DJ (2009) Lossof-function variants in endothelial lipase are a cause of elevated HDL cholesterol in humans. J Clin Invest 119(4):1042-1050. doi: $10.1172 / \mathrm{JCI} 37176$

36. Fujii H, Fukuda A, Tanaka M, Kojima Y, Ishida T, Hirata K, Fukagawa M (2008) Putative role of endothelial lipase in dialysis patients with hypoalbuminemia and inflammation. Am J Nephrol 28(6):974-981. doi:10.1159/000144025

37. Kardoulas DG, Katsamouris AN, Gallis PT, Philippides TP, Anagnostakos NK, Gorgoyannis DS, Gourtsoyannis NC (1996) Ultrasonographic and histologic characteristics of symptom-free and symptomatic carotid plaque. Cardiovasc Surg 4(5):580-590 (0967210996000300)

38. Carr SC, Cheanvechai V, Virmani R, Pearce WH (1997) Histology and clinical significance of the carotid atherosclerotic plaque: implications for endovascular treatment. J Endovasc Surg 4(4):321-325. doi:10.1583/1074-6218(1997)004<0321:HACSOT> 2.0.CO; 2

39. Papaspyridonos M, Smith A, Burnand KG, Taylor P, Padayachee S, Suckling KE, James CH, Greaves DR, Patel L (2006) Novel candidate genes in unstable areas of human atherosclerotic plaques. Arterioscler Thromb Vasc Biol 26(8):1837-1844. doi: 10.1161/01.ATV.0000229695.68416.76 